\title{
Moving beyond size: vorticity and energy loss are correlated with right ventricular dysfunction and exercise intolerance in repaired Tetralogy of Fallot
}

Yue-Hin Loke $1^{*}$ (D), Francesco Capuano ${ }^{2}$, Vincent Cleveland ${ }^{3}$, Jason G. Mandell ${ }^{1}$, Elias Balaras ${ }^{4}$ and Laura J. Olivieri ${ }^{1,3}$

\begin{abstract}
Background: The global effect of chronic pulmonary regurgitation (PR) on right ventricular (RV) dilation and dysfunction in repaired Tetralogy of Fallot (rTOF) patients is well studied by cardiovascular magnetic resonance (CMR). However, the links between PR in the RV outflow tract (RVOT), RV dysfunction and exercise intolerance are not clarified by conventional measurements. Not all patients with RV dilation share the same intracardiac flow characteristics, now measurable by time resolved three-dimensional phase contrast imaging (4D flow). In our study, we quantified regional vorticity and energy loss in rTOF patients and correlated these parameters with RV dysfunction and exercise capacity.

Methods: rTOF patients with 4D flow datasets were retrospectively analyzed, including those with transannular/ infundibular repair and conduit repair. Normal controls and RV dilation patients with atrial-level shunts (Qp:Qs > 1.2:1) were included for comparison. 4D flow was post-processed using IT Flow (Cardioflow, Japan). Systolic/diastolic vorticity $(\omega, 1 / s)$ and viscous energy loss (VEL, $\mathrm{mW}$ ) in the RVOT and RV inflow were measured. To characterize the relative influence of diastolic vorticity in the two regions, an RV Diastolic Vorticity Quotient ( $\omega_{\text {RVOT-Diastole }} / \omega_{\text {RV Inflow-Diastole, }}$ RV-DVQ) was calculated. Additionally, RVOT Vorticity Quotient ( $\omega_{\text {RVOT-Diastole }} / \omega_{\text {RVOT-Systole, }}$ RVOT-VQ) and RVOT Energy Quotient (VEL RVOT-Diastole $_{\text {NEL }}$ RVOT-Systole, RVOT-EQ) was calculated. In rTOF, measurements were correlated against conventional CMR and exercise stress test results.

Results: 58 rTOF patients, 28 RV dilation patients and 12 controls were included. RV-DVQ, RVOT-VQ, and RVOT-EQ were highest in rTOF patients with severe PR compared to rTOF patients with non-severe PR, RV dilation and controls $(p<0.001)$. RV-DVQ positively correlated with RV end-diastolic volume $(0.683, p<0.001)$, PR fraction $(0.774$, $p<0.001)$ and negatively with RV ejection fraction $(-0.521, p=0.003)$. Both RVOT-VQ, RVOT-EQ negatively correlated with $\mathrm{VO}_{2-\max }(-0.587, \mathrm{p}=0.008$ and $-0.617, \mathrm{p}=0.005)$ and $\%$ predicted $\mathrm{VO}_{2-\max }(-0.678, \mathrm{p}=0.016$ and -0.690 , $p=0.001)$.
\end{abstract}

\footnotetext{
${ }^{*}$ Correspondence: yloke@childrensnational.org

${ }^{1}$ Present Address: Division of Cardiology, Children's National Medical Center, 111 Michigan Ave NW, W3-200, Washington, DC 20010, USA

Full list of author information is available at the end of the article
}

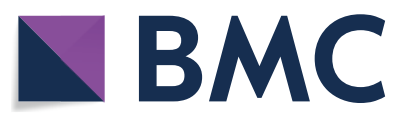

(c) The Author(s) 2021. Open Access This article is licensed under a Creative Commons Attribution 4.0 International License, which permits use, sharing, adaptation, distribution and reproduction in any medium or format, as long as you give appropriate credit to the original author(s) and the source, provide a link to the Creative Commons licence, and indicate if changes were made. The images or other third party material in this article are included in the article's Creative Commons licence, unless indicated otherwise in a credit line to the material. If material is not included in the article's Creative Commons licence and your intended use is not permitted by statutory regulation or exceeds the permitted use, you will need to obtain permission directly from the copyright holder. To view a copy of this licence, visit http://creativecommons.org/licenses/by/4.0/. The Creative Commons Public Domain Dedication waiver (http://creativeco mmons.org/publicdomain/zero/1.0/) applies to the data made available in this article, unless otherwise stated in a credit line to the data. 
Conclusions: In rTOF patients, vorticity and energy loss dominate the RVOT compared to tricuspid inflow, correlating with RV dysfunction and exercise intolerance. These 4D flow-based measurements may be sensitive biomarkers to guide surgical management of rTOF patients.

Keywords: Tetralogy of Fallot, Intracardiac flow, Vorticity, 4D flow

\section{Background}

In repaired Tetralogy of Fallot (rTOF) patients, the longterm consequences of chronic pulmonary regurgitation (PR) on right ventricular (RV) dilation, RV dysfunction, ventricular arrhythmias and mortality have been wellstudied [1]. Thus, measurements of RV size and function as guided by cardiovascular magnetic resonance (CMR) have provided critical interventional thresholds for pulmonary valve replacement (PVR) [2,3]. However, current guidelines acknowledge the limitations of these conventional CMR measurements [2,3], as they do not necessarily predict deterioration of the RV [4], nor do they incorporate the diversity of RV morphology, RV motion, and RV outflow tract (RVOT) geometry [5-7]. Exclusive interpretation of the RV in rTOF using indexed RV end-diastolic volume (RVEDVI), indexed RV endsystolic volume (RVESVI), RV ejection fraction (RVEF) and pulmonary regurgitant fraction (PR\%) does not fully describe RV dysfunction, nor correlate with exercise intolerance [8-11]. Furthermore, the direct link between PR, RV dysfunction and exercise intolerance has not been clarified, as the resultant intracardiac flow abnormalities from PR have not been comprehensively studied in rTOF patients.

Recent advances in CMR now allow for time-resolved three-dimensional phase contrast imaging (4D flow) of intracardiac flow phenomena. With 4D flow, it is observed that intracardiac flow parameters such as vorticity (the local spinning motion of blood) and kinetic energy are altered in rTOF patients [12-15]. Intracardiac flow parameters are thus promising hemodynamic biomarkers to potentially detect RV dysfunction, although these studies have mainly correlated 4D flow biomarkers with PR and RV dilation. These studies have also focused on global quantification of the RV, without considering regional abnormalities created from PR in the RVOT. There have also not been any 4D flow studies that have compared rTOF against other forms of chronic volume loading in the RV.

Beyond size, dilated RVs do not all share the same intracardiac flow characteristics. Both in-vivo and in-vitro studies have highlighted the unique pathophysiology of rTOF patients [16-20] that are fundamentally distinct from other forms of RV dilation. A recent echocardiographic case-control study by Moceri et al. [17] demonstrated different RV strain patterns between rTOF and patients with atrial septal defects (ASD), despite similar RV size. We have previously developed an in-vitro computational model of intracardiac flow in rTOF patients using conventional CMR imaging [18-20]. Through this computational model, we observed a pattern where the jet of PR disrupts the natural tricuspid inflow vortex at the RVOT, a finding also observed in an in-vitro pump study by Mikhail et al. [16]. We conjecture that in rTOF patients this flow phenomenon can be quantified by $4 \mathrm{D}$ flow as patterns of vorticity and energy loss that are distinct from the normal RV and RV dilation patients.

Thus, in our study, we quantified regional vorticity and energy loss in rTOF patients, and compared these values to normal controls and RV dilation patients (those with dilated RVs from atrial level shunts). We hypothesized that in rTOF patients, there is distinct influence of vorticity and energy loss in the RVOT compared to normal control and RV dilation patients, and that these intracardiac flow parameters are correlated with RV dysfunction and exercise intolerance.

\section{Methods}

This was an Institutional Review Board-approved retrospective study involving patients who underwent a CMR study between December 2018 and August 2020. rTOF patients included those with pulmonary stenosis (TOFPS) or double-outlet right ventricle (DORV) variants who underwent either transannular or infundibular patch repair, as well as those with pulmonary atresia (TOF-PA) who underwent RV-to-pulmonary artery (RV-PA) conduit repair. Patients with poor imaging quality or significant stent/sternal artifact were excluded. Patients with evidence of elevated pulmonary vascular resistance (confirmed by cardiac catheterization) were also excluded. For comparison, CMR datasets from normal controls and RV dilation patients were included. RV dilation patients were defined as those with ASDs or partial anomalous pulmonary venous return (PAPVR) with hemodynamically significant shunt (Qp:Qs>1.2:1). Normal controls included those who underwent CMR imaging for separate clinical indication (exclude cardiomyopathy, atrial shunts or PAPVR) and found to have normal RV size, function and pulmonary-to-systemic flow ratio (Qp:Qs) < 1.2:1.

All studies were performed with a $1.5 \mathrm{~T}$ CMR scanner (Siemens Healthineers, Erlangen, German). CMR data included cine imaging (long-axis and short-axis 
cine), contrast-enhanced magnetic resonance angiography (MRA), three-dimensional (3D) steady state free precession imaging (3D SSFP), two-dimensional phase contrast across the pulmonary valve (Venc set between 2 and $2.5 \mathrm{~m} / \mathrm{s}$ ) and 4D flow. The 4D flow sequence parameters included FOV $=280-480 \times 140-230 \mathrm{~mm}$, matrix $=160 \times 77, \quad \mathrm{TE}=2.19 \mathrm{~ms}, \quad \mathrm{TR}=37.9-59.4 \mathrm{~ms}$ (dependent on number of segments per RR and RR interval; either 2 segments for $R R>750$ ms or 3 segments for $R R<750 \mathrm{~ms}$ ), flip angle $=15$, slice thickness $=1.8 \mathrm{~mm}$ or $2.8 \mathrm{~mm}$ (dependent on patient size, either $1.8 \mathrm{~mm}$ for BSA $<1.5 \mathrm{~m}^{2} ; 2.8 \mathrm{~mm}$ for $\mathrm{BSA}>1.5 \mathrm{~m}^{2}$ ), venc $=2 \mathrm{~m} / \mathrm{s}-$ $2.5 \mathrm{~m} / \mathrm{s}$ and number of reconstructed phases $=20-30$. The MRA and 3D SSFP covered the entire heart with voxel size $\sim 1.4 \times 1.4 \times 1.4 \mathrm{~mm}$. Standard clinical measurements of RVEDVI, RVESVI, RVEF, and PR\% were obtained. For rTOF patients, electrocardiogram and cardiopulmonary exercise stress test results within 1 year of CMR study were also collated for QRS duration, $\mathrm{VO}_{2-\max }$ and $\%$ predicted $\mathrm{VO}_{2-\max }$.

\section{D modeling of RVOT and RV inflow}

3D end-diastolic models of the RV were first created from MRA and 3D SSFP datasets, according to lab standard segmentation [21, 22] using commercially available software (Mimics; Materialise, Leuven, Netherlands). The RV was isolated between the tricuspid valve and pulmonary valve annuli. For rTOF with transannular patch, the pulmonary valve annulus was determined by presence of either residual valve sinuses or valve tissue. The 3D models were then consistently divided into separate components of the RV (Fig. 1), accounting for the variable geometry of the RV and RVOT: \#1) A dividing plane (parallel to 4-chamber cine plane) at the antero-septal commissure of tricuspid annulus (most cranial aspect) was first used to separate the RVOT and RV body; \#2) the remaining RV body was then divided half-way down the long-axis to separate the RV inflow from RV apex. The resulting RVOT volume is in line with corresponding RV segments as described by Wald et al. (which also separated the RV into components) [23].
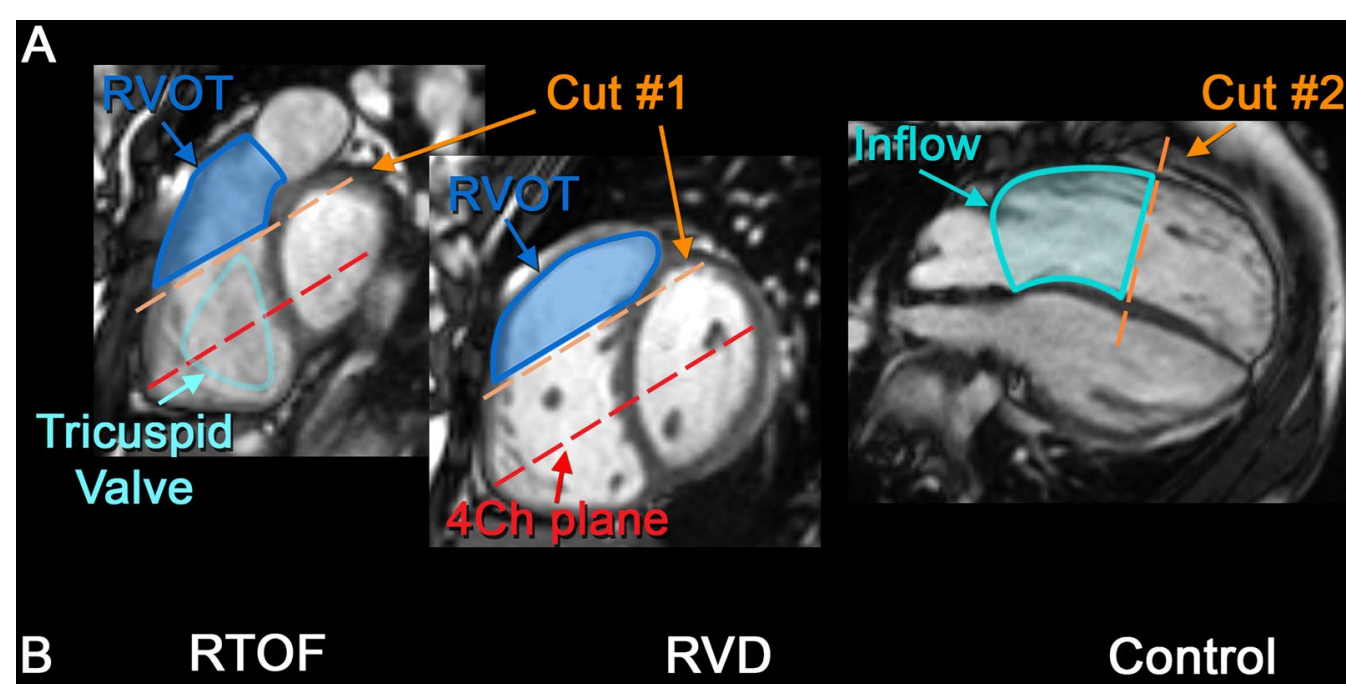

RVD

Control

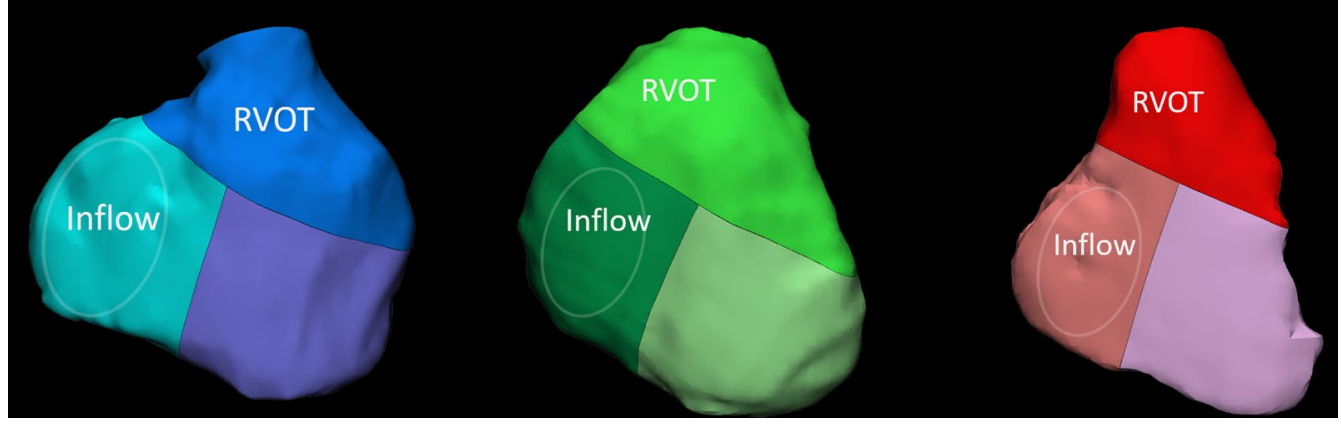

Fig. 1 The inflow and outflow components of the right ventricle (RV). Three-dimensional (3D) end-diastolic models of the RV for all three groups were systematically performed to separate out the RV outflow tract (RVOT) and RV inflow. This was performed by Cut \#1 and Cut \#2 as shown in $\mathbf{A}$ with corresponding landmarks. The resulting 3D models of the inflow and outflow components are shown in $\mathbf{B}$ 


\section{Post-processing of 4D flow}

Each 4D flow dataset was imported into IT Flow (Cardioflow, Tokyo, Japan) [24] as separate segmentation masks to isolate velocity flow fields within the domains of interest. After background-phase correction, the masks were further screened to remove regions of noise artifact. Particle tracking and vorticity mapping analysis was also performed for qualitative assessment.

\section{Quantification of vorticity, RVOT vorticity quotient and RV diastolic vorticity quotient}

Vorticity was quantified for each phase of the cardiac cycle by IT Flow. Conceptually, vorticity represents the magnitude and the axial direction of spinning. Vorticity is defined as the curl of the velocity field:

$$
\vec{\omega}=\nabla \times \vec{u}
$$

Absolute vorticity (units of L/s) is calculated by spatially integrating the vorticity magnitude $\|\vec{\omega}\|$, that accounts for all three directions of spinning, within each voxel:

$$
\text { Absolute Vorticity }=\int_{V}\|\vec{\omega}\| d V
$$

where $\mathrm{V}$ is the segmented volume of interest (e.g. RVOT or inflow region). Since absolute vorticity is dependent on segmentation volume (units of $\mathrm{L}$ ), the calculated results were normalized against the volume of the segmented region as $\omega$ (units of $1 / \mathrm{s}$ ):

$$
\begin{gathered}
\omega_{R V O T}=\frac{\text { Absolute Vorticity }_{R V O T}}{\text { Volume }_{R V O T}} \\
\omega_{R V \text { Inflow }}=\frac{\text { Absolute Vorticity }_{\text {RVInflow }}}{\text { Volume }_{\text {RVInflow }}}
\end{gathered}
$$

The peak value within the RVOT during systole $\left(\omega_{\text {RVOT-Systole }}\right)$, as well as the RVOT and RV inflow during diastole $\left(\omega_{\text {RVOT-Diastole, }} \omega_{\mathrm{RV}}\right.$ Inflow-Diastole $)$ were
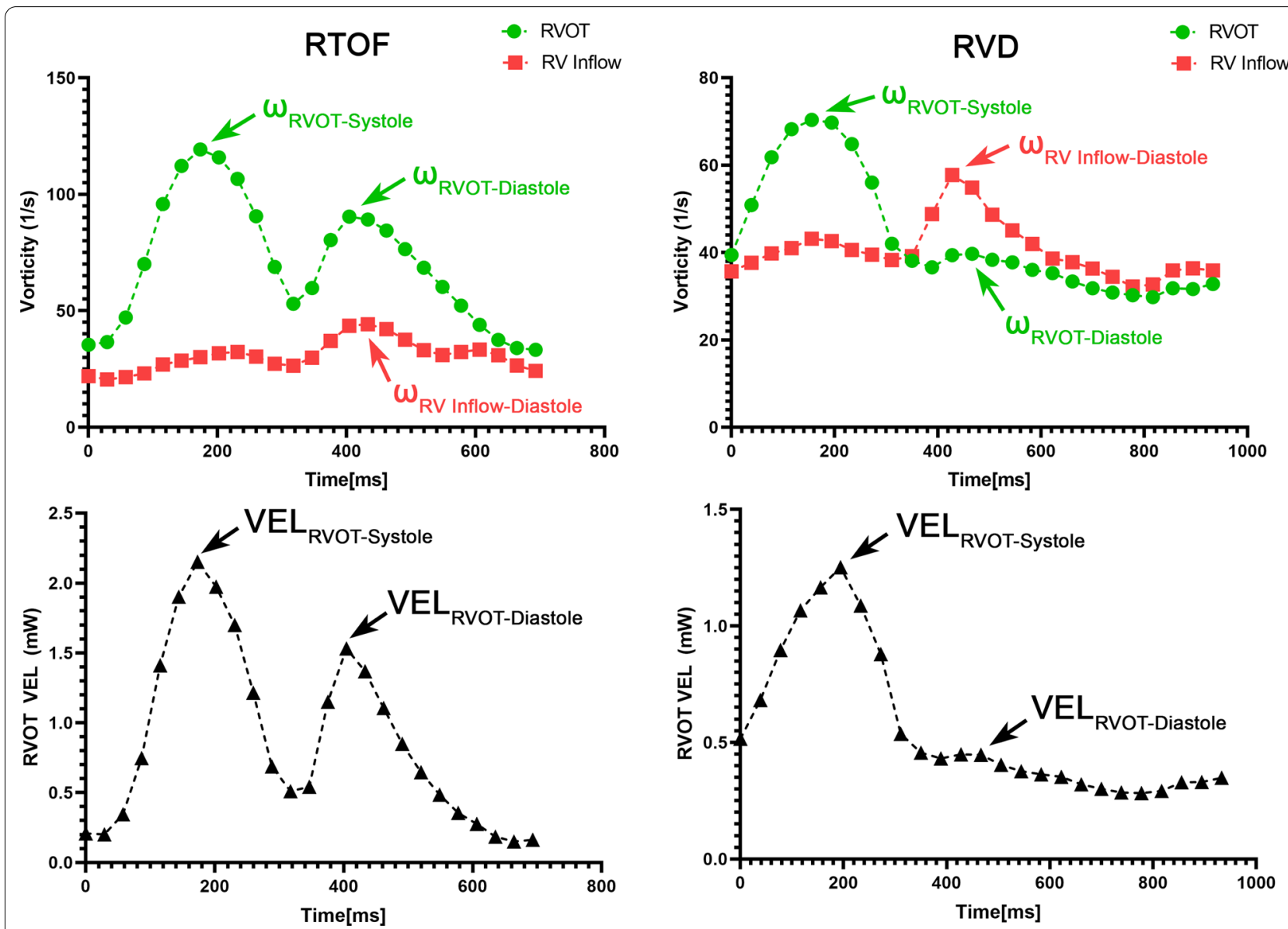

Fig. 2 Example vorticity and energy loss profiles in a repaired tetralogy of Fallot (rTOF) and right ventricular dilation (RVD) patient. For each dataset, the peak systolic/diastolic viscous energy loss, systolic/diastolic vorticity in the RV outflow tract (RVOT) and RV inflow was selected 
measured (Fig. 2). To characterize the relative influence of vorticity between RV inflow vs PR, and remove other confounding effects such as heart rate and voxel size [25], these measurements were normalized into unitless parameters in a similar manner as PR\% and echocardiographic Doppler metrics of rTOF [26, 27]. $\omega_{\text {RVOT-Diastole }} /$ $\omega_{\mathrm{RV}}$ Inflow-Diastole was calculated and represented as the RV Diastolic Vorticity Quotient (RV-DVQ). $\omega_{\text {RVOT- }}$ Diastole $/ \omega_{\text {RVOT-Systole }}$ was also calculated and represented as the RVOT Vorticity Quotient (RVOT-VQ).

\section{Quantification and analysis of RVOT viscous energy loss, RVOT energy quotient}

Viscous energy loss (VEL, $\mathrm{mW}$ ) represent viscous dissipation across the flow field within each voxel, a combined effect of blood viscosity and velocity gradient (shear) [24]. For each direction, E is summated over repeated indices as:

$$
V E L=\int \frac{1}{2} \mu \sum\left(\frac{\partial u_{i}}{\partial x_{j}}+\frac{\partial u_{j}}{\partial x_{i}}\right)^{2} d V
$$

where $\mu$ is blood viscosity at $0.004 \mathrm{~Pa} \cdot \mathrm{s}$. The peak VEL across the RVOT in systole $\left(\mathrm{VEL}_{\mathrm{RVOT}-\text {-Systole }}\right)$ and diastole (VEL RVOT-Diastole) was measured (Fig. 2). VEL RVOT-Diastole $_{\text {f }}$ ) $\mathrm{VEL}_{\mathrm{RVOT}-\text { Systole }}$ was also calculated as the RVOT Energy Quotient (RVOT-EQ).

\section{Statistical analysis}

All statistical analysis was performed with Prism 8 (Graphpad, San Diego, California, USA). One-way analysis of variance (ANOVA) was used to compare between rTOF patients with severe PR (defined as PR\% $>40 \%$ ), rTOF patients with moderate PR (PR\% 20-40\%), rTOF patients with mild PR (PR\%<20\%), RV dilation and control groups. Subgroup analysis of rTOF patients was also performed: \#1) rTOF patients with TOF-PS anatomy and only infundibular or transannular patch repair (i.e. no conduit placement) with no further surgical reintervention (i.e. no PVR), reducing any variation to RVOT geometry from a conduit hood or PVR; \#2) rTOF patients with exercise stress test results. Correlations between continuous variables were assessed using Pearson's correlation coefficient [28]. Probability values $<0.05$ were considered statistically significant.

\section{Results \\ Demographics}

58 rTOF patients, 28 RV dilation patients and 12 normal controls were included (Table 1). rTOF patients consisted of 47 with TOF-PS (2 DORV variants) and 11 with TOF-PA; 42 had transannular patch repair, 7 had infundibular patch repair, and 9 underwent RV-PA conduit repair. 11 rTOF patients were statuspost PVR at the time of CMR (Table 2). The mean PR\% was $27.9 \pm 16.4 \%$, with 20 patients having severe PR, 18 with moderate PR and 20 with mild PR. RV

Table 1 Overall demographics

\begin{tabular}{|c|c|c|c|c|c|}
\hline & rTOF patients $(n=58)$ & $\begin{array}{l}\text { RV dilation patients } \\
(n=28)\end{array}$ & $p$ & Normal controls $(n=12)$ & $p$ \\
\hline \multicolumn{6}{|l|}{ Demographics } \\
\hline Age (years), IQR & $21.7(12.2-29.6)$ & $11.5(4.0-15.5)$ & $<0.001$ & $13.9(9.1-17.7)$ & 0.038 \\
\hline Female & $30(51 \%)$ & $14(50 \%)$ & & $6(50 \%)$ & \\
\hline $\mathrm{BSA}\left(\mathrm{m}^{2}\right)$ & $1.5 \pm 0.40$ & $1.2 \pm 0.6$ & $<0.001$ & $1.4 \pm 0.5$ & ns \\
\hline \multicolumn{6}{|l|}{ Native anatomy } \\
\hline & $\begin{array}{l}\text { TOF-PS } 47(84 \%) \\
\text { TOF-PA } 11(16 \%)\end{array}$ & $\begin{array}{l}\text { PAPVR } 24 \text { (85\%) } \\
2^{\circ} \text { ASD } 4 \text { (15\%) }\end{array}$ & & & \\
\hline \multicolumn{6}{|l|}{ CMR data } \\
\hline RVEDV $(m L)$ & $203 \pm 81$ & $165 \pm 110$ & ns & $131 \pm 55$ & 0.0017 \\
\hline RVEDVI $\left(\mathrm{mL} / \mathrm{m}^{2}\right)$ & $131 \pm 33$ & $148 \pm 57$ & ns & $86 \pm 15$ & $<0.001$ \\
\hline RVESVI $\left(\mathrm{mL} / \mathrm{m}^{2}\right)$ & $65 \pm 21$ & $61 \pm 29$ & ns & $38 \pm 10$ & $<0.001$ \\
\hline RVEF (\%) & $51.1 \% \pm 5.5 \%$ & $60.4 \% \pm 6.1 \%$ & $<0.001$ & $60.0 \% \pm 5.8 \%$ & $<0.001$ \\
\hline PR\% & $27.9 \% \pm 16.4 \%$ & $0.0 \pm 0.04 \%$ & $<0.001$ & $0.0 \pm 0.03 \%$ & $<0.001$ \\
\hline Qp:Qs & $1.0 \pm 0.05$ & $2.5 \pm 1.4$ & $<0.001$ & $1.0 \pm 0.08$ & ns \\
\hline
\end{tabular}

Fifty-eight repaired tetralogy of Fallot (rTOF) patients, 28 patients with right ventricular (RV) dilation and twelve normal controls were included. RV dilation patients had either partial anomalous pulmonary venous return (PAPVR) or large secundum type atrial septal defects ( $2^{\circ}$ ASD). There was selection bias in that rTOF patients tended to be older and larger than RV dilation patients, although had similar indexed right ventricular end-diastolic volume index (RVEDVI) and RV end-systolic volume index (RVESVI)

$B S A$ body surface area, RVEF right ventricular ejection fraction, $P R \%$ pulmonary regurgitation percent 
Table 2 Demographics of repaired tetralogy of Fallot (rTOF) cohort

\begin{tabular}{llc}
\hline & TOF-PS $(\mathbf{n}=\mathbf{4 7})$ & TOF-PA ( $\mathbf{n}=\mathbf{1 1})$ \\
\hline Age of complete repair (years), IQR & $1.4(0.3-1.9)$ & $1.2(0.2-1.7)$ \\
Type of surgery & & $3(27 \%)$ \\
$\quad$ Initial Blalock-Taussig-Thomas shunt palliation & $8(17 \%)$ & $3(27 \%)$ \\
Transannular patch & $39(83 \%)$ & $0(0 \%)$ \\
Infundibular patch & $7(15 \%)$ & $8(72 \%)$ \\
Right ventricle-to-pulmonary artery conduit & $1(2 \%)$ & 2 by surgery, 1 by \\
Subsequent pulmonary valve replacement & 8 by surgery (17\%) & catheterization \\
& & $(27 \%)$ \\
\hline
\end{tabular}

The cohort consisted of patients with either tetralogy of Fallot-Pulmonary Stenosis (TOF-PS) or tetralogy of Fallot-Pulmonary Atresia (TOF-PA). 10 patients already had pulmonary valve replacement

dilation patients consisted of 24 with PAPVR, and 4 with secundum type ASD. Within PAPVR patients, 17 had right-sided PAPVR and associated atrial communication (15 with superior sinus venosus defect, 2 with inferior sinus venosus defect), and the remaining 7 had isolated left-sided PAPVR. There was selection bias in that rTOF patients were older and larger compared to RV dilation and control patients, although both rTOF and RV dilation patients had similar enlarged RVEDV and RVEDVI $\left(203 \pm 81 \mathrm{~mL}\right.$ and $131 \pm 33 \mathrm{~mL} / \mathrm{m}^{2}$ vs $165 \pm 110 \mathrm{~mL}$ and $\left.147 \pm 58 \mathrm{~mL} / \mathrm{m}^{2}, \mathrm{p}=\mathrm{ns}\right)$.

\section{Comparison of vorticity between rTOF, RV dilation and control}

Overall quantification results of vorticity are shown in Fig. 3A, B. Between rTOF, RV dilation and control groups, there were no significant differences in regional systolic vorticity $(\mathrm{p}=\mathrm{ns})$. Meanwhile, $\omega_{\mathrm{RVOT}}$ Diastole, $\omega_{\mathrm{RV}}$ Inflow-Diastole, RVOT-VQ and RV-DVQ were different across groups $(\mathrm{p}<0.001)$. $\omega_{\text {RVOT-Diastole }}$ and RVOT-VQ were highest in rTOF with severe PR $(96.5 \pm 15.6 / \mathrm{s}, 1.05 \pm 0.26)$ compared to rTOF with moderate PR $(75.3 \pm 15.3 / \mathrm{s}, 0.73 \pm 0.21)$, rTOF with mild PR $(57.6 \pm 15.9 / \mathrm{s}, 0.70 \pm 0.20)$, RV dilation $(64.7 \pm 21.5 / \mathrm{s}, 0.75 \pm 0.28)$ and normal controls $(50.2 \pm 16.9 / \mathrm{s}, 0.56 \pm 0.18) . \omega_{\text {Inflow-Diastole }}$ was highest in RV dilation patients $(77.9 \pm 25.3 / \mathrm{s})$, compared to rTOF patient with severe PR $(52.7 \pm 13.6 / \mathrm{s})$, rTOF patients with moderate PR $(54.5 \pm 12.0 / \mathrm{s})$, rTOF with mild PR $(50.4 \pm 8.6 / \mathrm{s})$, and control group $(58.9 \pm 17.8 / \mathrm{s})$. RVDVQ was highest in rTOF with severe PR $(1.88 \pm 0.33)$, compared to rTOF with moderate PR $(1.43 \pm 0.34)$ and rTOF with mild PR (1.17 \pm 0.43$)$; whereas RV-DVQ is normalized in RV dilation patients $(0.85 \pm 0.18)$ and similar to normal controls $(0.87 \pm 0.26)$.

\section{Comparison of RVOT energy loss between rTOF, RV dilation and control}

VEL measurements across the RVOT are demonstrated in Fig. 3C. There were no significant differences in VEL $\mathrm{RVOT}_{\text {-Systole }}$ between $\mathrm{rTOF}$, RV dilation and control groups $(\mathrm{p}=\mathrm{ns})$. Meanwhile, there were differences in VEL $\mathrm{RVOT}_{\text {-Diastole }}$ and RVOT-EQ $(\mathrm{p}<0.001)$. $V_{\text {VELOT-Diastole }}$ and RVOT-EQ was highest in $\mathrm{rTOF}$ with severe PR $(2.53 \pm 1.51 \mathrm{~mW}, 1.24 \pm 0.65)$ compared to rTOF with moderate PR $(1.18 \pm 1.03 \mathrm{~mW}, 0.56 \pm 0.31)$, rTOF with mild PR $(1.27 \pm 1.31 \mathrm{~mW}, 0.55 \pm 0.24)$, RV dilation patients $(1.12 \pm 1.16 \mathrm{~mW}, 0.75 \pm 0.28)$ and normal controls $(0.50 \pm 0.58 \mathrm{~mW}, 0.56 \pm 0.18)$.

\section{Qualitative comparison of intracardiac flow}

The representative differences in flow topology between each cohort are demonstrated by velocity vector fields (Fig. 4), particle tracking (Fig. 5A) and vorticity mapping (Fig. 5B). During systole, the particle tracking profile appeared similar between groups. In diastole for both RV dilation and control cases, an organized, "donut"-shaped ring-vortex was visualized surrounding the tricuspid inflow. The superior edge of the diastolic vortex propagated in a counterclockwise pattern directly towards the RVOT. In comparison, the rTOF cohort in diastole demonstrated dominant vorticity formation starting in the RVOT from PR, limiting the ring-vortex profile from tricuspid valve.

\section{Subgroup analysis of TOF-PS patients and only primary repair}

Among the rTOF cohort, there were 36 patients who had TOF-PS status-post infundibular or transannular patch repair with no further surgical reintervention. Within this subgroup, there were fair-to-high correlations between 
A Systole-RVOT

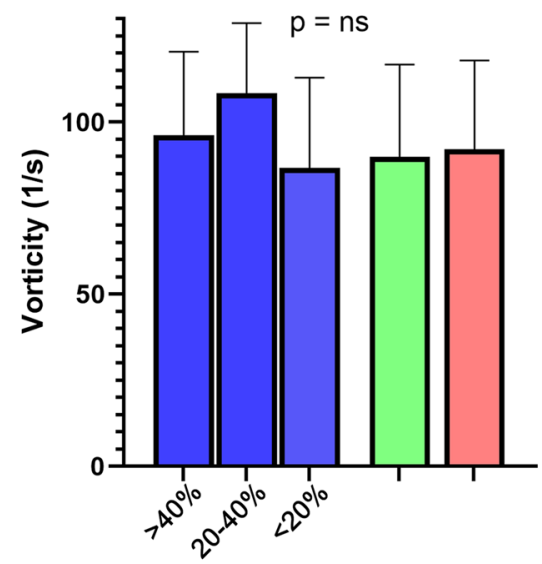

B

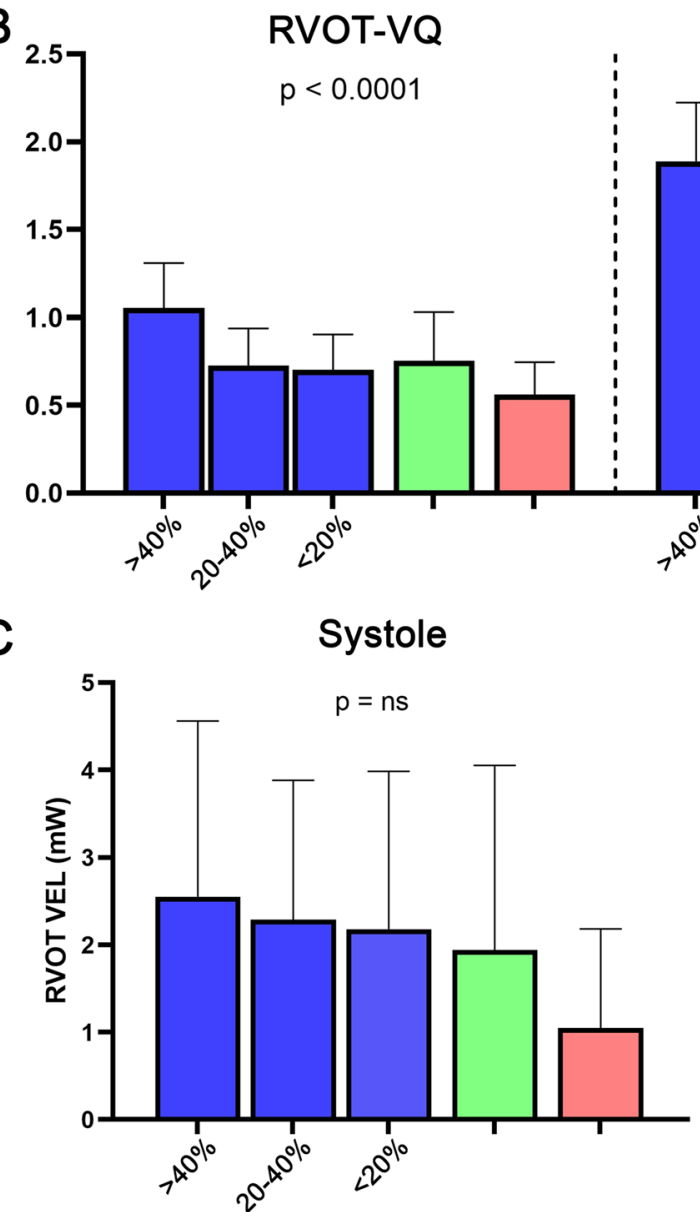

Diastole-RVOT

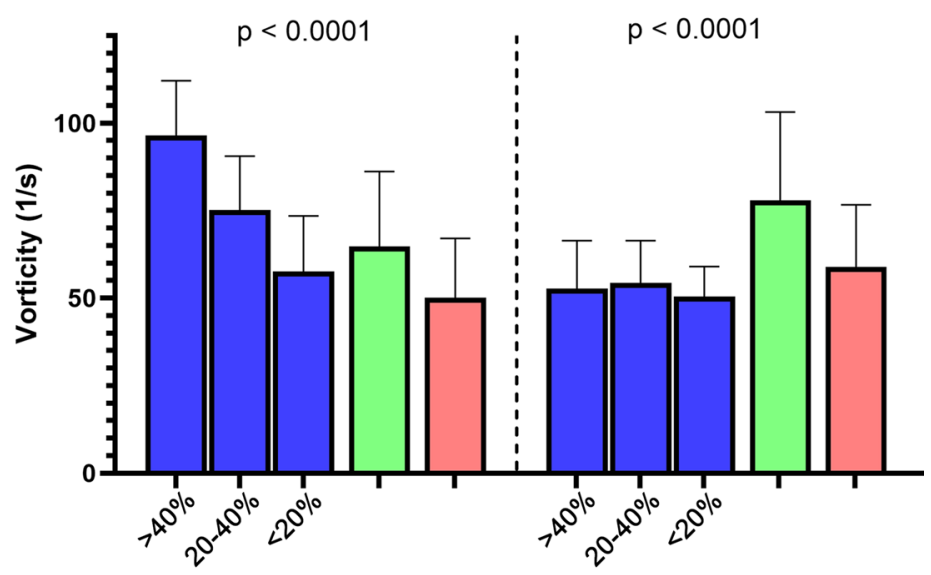

RV-DVQ

$p<0.0001$
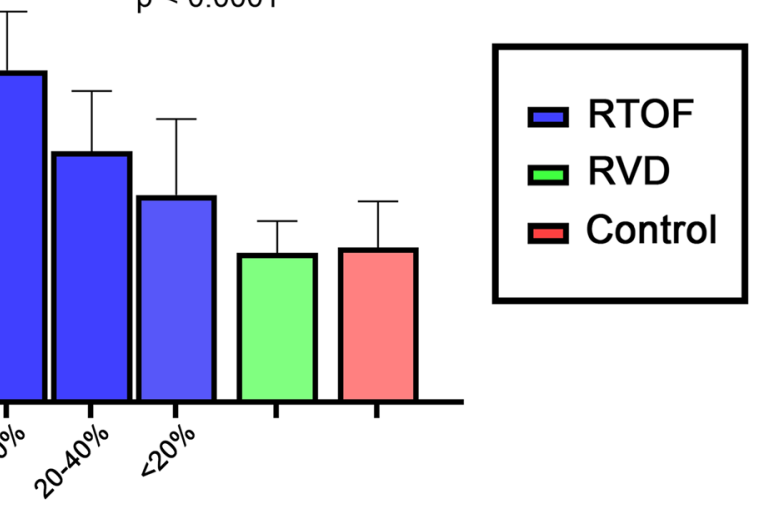

Diastole

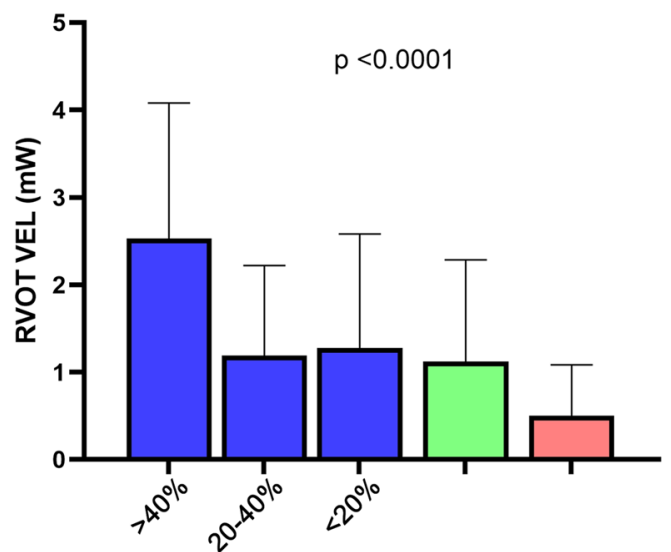

Fig. 3 Vorticity and power loss results. When compared to right ventricular dilation (RVD) and control groups, repaired tetralogy of Fallot (rTOF) patients with severe pulmonary regurgitation (PR\% > 40\%) had the highest diastolic vorticity in the right ventricular outflow tract (RVOT), as shown in $\mathbf{A}$. This group also had the highest diastolic vorticity quotient (RV-DVQ) and right ventricular outflow tract vorticity quotient (RVOT-VQ) as shown in $\mathbf{B}$; as well as the highest RVOT viscous energy loss (VEL) as shown in $\mathbf{C}$

intracardiac flow parameters with RVEDVI, RVESVI, RVEF and PR\% (Table 3, Fig. 5A-C). RV-DVQ correlated with RVEF $(-0.521, \mathrm{p}=0.001), \mathrm{PR} \%(0.774, \mathrm{p}<0.001)$,
RVEDVI (0.683, $\mathrm{p}<0.001)$, RVESVI $(0.696, \mathrm{p}<0.001)$ and QRS duration $(0.402, \mathrm{p}=0.017)$. RTOF-EQ correlated 


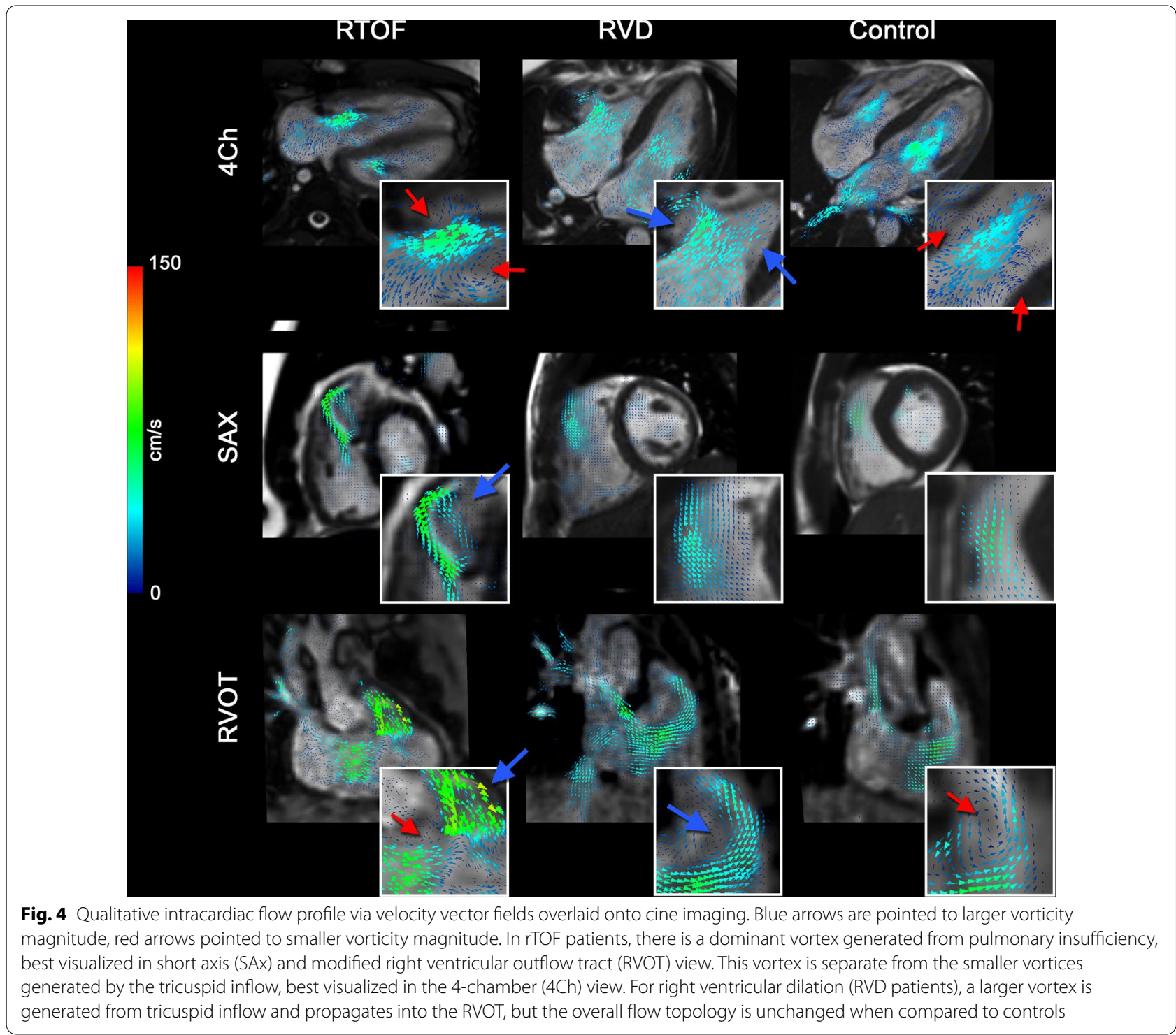

with RVEF $(-0.472, \mathrm{p}=0.0032), \mathrm{PR} \%(0.584, \mathrm{p}<0.001)$, RVEDVI (0.415, $\mathrm{p}=0.011)$, RVESVI $(0.461, \mathrm{p}=0.004)$.

\section{Subgroup analysis of rTOF patients with exercise stress test} Among the rTOF cohort, 19 patients had exercise stress test performed within $2.1 \pm 5.4$ months of CMR study. This subgroup cohort consisted of 16 with TOF-PS and 3 with TOF-PA; 17 had transannular patch, 2 had conduit repair. 7 patients already had PVR. The mean $\mathrm{PR} \%$ was $30 \pm 16 \%$. The mean $\mathrm{VO}_{2 \text {-max }}$ and \% predicted $\mathrm{VO}_{2-\max }$ was $27.4 \pm 7.72 \mathrm{~mL} / \mathrm{kg} / \mathrm{min}$ and $73.6 \pm 23.5 \%$ respectively. There were no significant correlations between $\mathrm{VO}_{2-\max }$ and \% predicted $\mathrm{VO}_{2-\max }$ with conventional CMR measurements of the RV. Meanwhile, exercise stress test results moderately correlated with 4D flow parameters (Table 4, Fig. 6D). RVOTVQ and RVOT-EQ negatively correlated with $\mathrm{VO}_{2-\max }$ $(-0.587, \mathrm{p}=0.008 ;-0.617, \mathrm{p}=0.005)$ and $\%$ predicted $\mathrm{VO}_{2-\max }(-0.678, \mathrm{p}=0.0014 ;-0.690, \mathrm{p}=0.001)$.

\section{Discussion}

This study retrospectively analyzed 4D flow datasets to quantify regional vorticity and energy loss in rTOF patients, compared to normal normal controls and RV dilation patients. The main findings of this study include: (1) increased RVOT vorticity and energy loss in diastole 


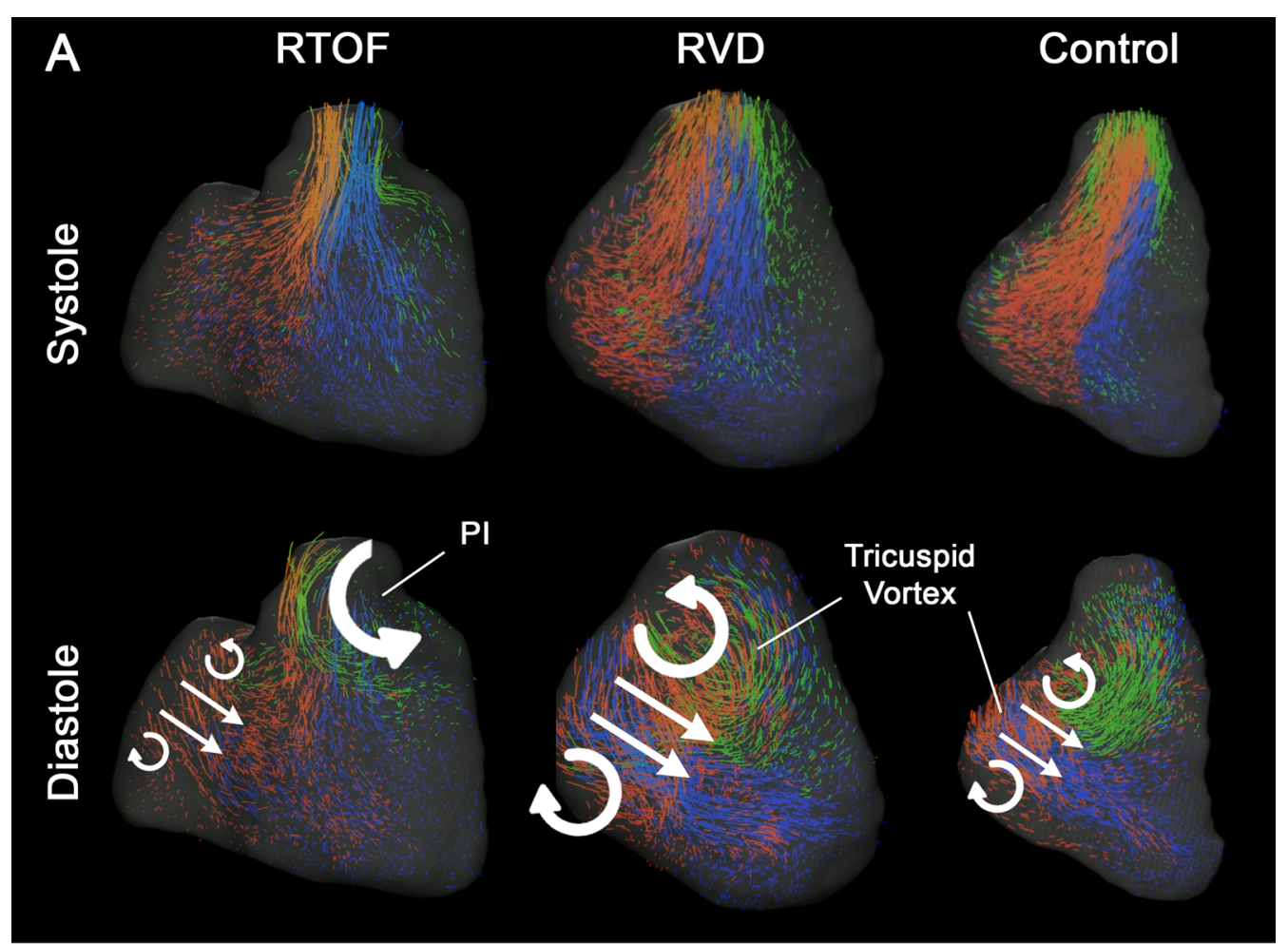

B $\quad \mathrm{RV}-\mathrm{DVQ}=\boldsymbol{\omega}_{\mathrm{RVOT} \text {-Diastole }} / \omega_{\mathrm{RV} \text { Inflow-Diastole }}$

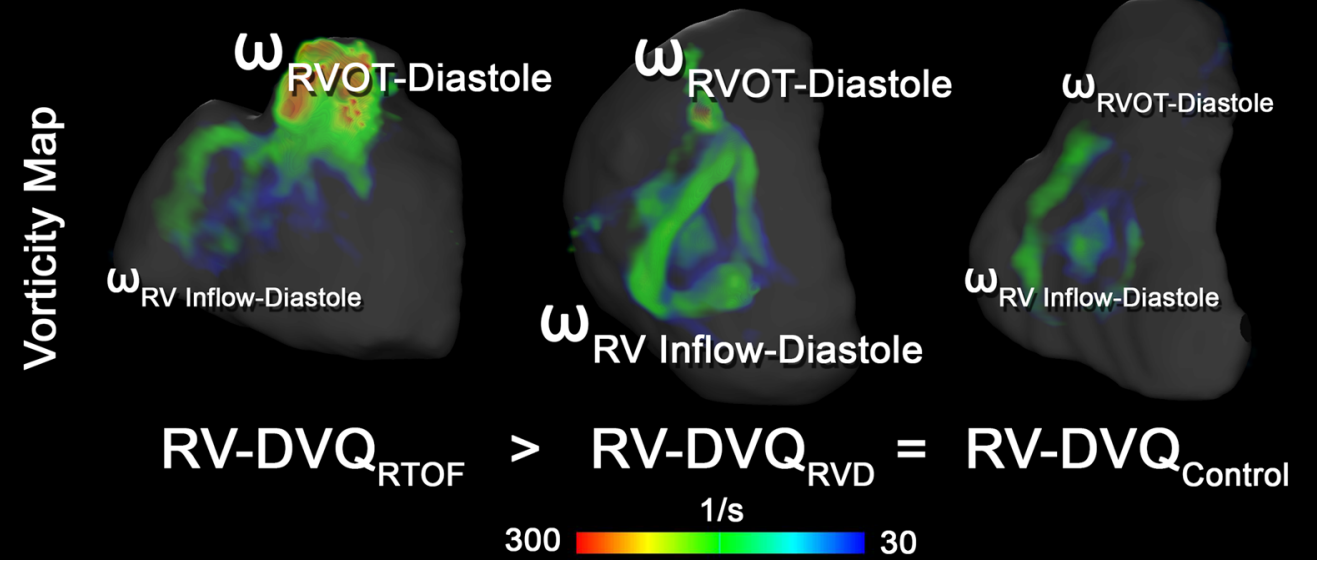

Fig. 5 Qualitative intracardiac flow profile via particle tracking (A, B) vorticity mapping. A Particles were color-coded based on original position in end-diastole. Red particles start from tricuspid inflow, green particles from right ventricular outflow tract (RVOT), and blue from remaining body/ apex. An organized, "donut"-shaped ring-vortex was visualized surrounding the tricuspid inflow in right ventricular dilation (RVD) and control cases, whereas this profile is disrupted in repaired tetralogy of Fallot (rTOF) patients. B Volumetric representation of vorticity also demonstrates the ring-vortex as shown. The calculation of RV Diastolic Vorticity Quotient (RV-DVQ) in RTOF patients demonstrates the influence of vorticity from RVOT; whereas in RVD and control cases, vorticity from RV inflow remains dominant

in rTOF patients, whereas diastolic vorticity in RV inflow remained dominant in normal controls and RV dilation patients; (2) correlation of RVOT vorticity and energy loss with RV dysfunction in TOF-PS patients; and (3) correlation of RVOT vorticity and energy loss with exercise intolerance.

Conventional CMR measurements inform the timing of PVR, albeit with limitations. The timing of PVR should 
Table 3 Subgroup correlation analysis of repaired tetralogy of Fallot (rTOF) patients who had infundibular or transannular patch repair (i.e. no conduit placement) and no pulmonary valve replacement $(n=36)$

\begin{tabular}{|c|c|c|c|c|c|c|c|c|c|c|}
\hline & RVEF \% & $p$ & PR \% & $p$ & RVEDVI & $p$ & RVESVI & $p$ & QRS & $\mathbf{P}$ \\
\hline \multicolumn{11}{|l|}{ Conventional } \\
\hline RVEF (\%) & & & -0.460 & 0.0048 & -0.453 & 0.005 & -0.685 & $<0.001$ & -0.408 & 0.015 \\
\hline PR\% & -0.460 & 0.005 & & & 0.702 & $<0.001$ & 0.695 & $<0.001$ & 0.408 & 0.017 \\
\hline RVEDVI $\left(\mathrm{mL} / \mathrm{m}^{2}\right)$ & -0.453 & 0.005 & 0.702 & $<0.001$ & & & 0.956 & $<0.001$ & 0.421 & 0.012 \\
\hline RVESVI $\left(\mathrm{mL} / \mathrm{m}^{2}\right)$ & -0.685 & $<0.001$ & 0.695 & $<0.001$ & 0.956 & $<0.001$ & & & 0.449 & 0.007 \\
\hline QRS (ms) & -0.4075 & 0.015 & 0.4076 & 0.017 & 0.4211 & 0.012 & 0.4498 & 0.007 & & \\
\hline \multicolumn{11}{|l|}{ Vorticity } \\
\hline$\omega_{\text {RVOT-Diastole }}$ & -0.327 & 0.048 & 0.794 & $<0.001$ & 0.637 & $<0.001$ & 0.599 & $<0.001$ & 0.247 & ns \\
\hline RTOF-VQ ( $\left.\omega_{\text {RVOT-Diastole }} / \omega_{\text {RVOT-Systole }}\right)$ & -0.496 & 0.002 & 0.7390 & $<0.001$ & 0.635 & $<0.001$ & 0.644 & $<0.001$ & 0.313 & ns \\
\hline RV-DVQ ( $\left.\omega_{\text {RVOT-Diastole }} / \omega_{\text {RV Inflow-Diastole }}\right)$ & -0.521 & 0.001 & 0.774 & $<0.001$ & 0.683 & $<0.001$ & 0.696 & $<0.001$ & 0.402 & 0.017 \\
\hline \multicolumn{11}{|l|}{ Energy loss } \\
\hline VEL $L_{\text {RVOT-Diastole }}$ & -0.373 & 0.023 & 0.607 & $<0.001$ & 0.747 & $<0.001$ & 0.721 & $<0.001$ & 0.392 & 0.020 \\
\hline RTOF-EQ (VEL & -0.472 & 0.003 & 0.584 & $<0.001$ & 0.415 & 0.011 & 0.461 & 0.004 & 0.201 & ns \\
\hline
\end{tabular}

Table 4 Subgroup correlation analysis of repaired retralogy of Fallot (rTOF) patients with exercise stress test results $(n=19)$

\begin{tabular}{|c|c|c|c|c|}
\hline & $\mathrm{VO}_{2-\max }$ & p & $\%$ Predicted $\mathrm{VO}_{2-\max }$ & p \\
\hline \multicolumn{5}{|l|}{ Conventional } \\
\hline RVEF (\%) & -0.291 & ns & 0.259 & ns \\
\hline PR\% & -0.192 & ns & -0.267 & ns \\
\hline RVEDVI $\left(\mathrm{mL} / \mathrm{m}^{2}\right)$ & 0.252 & ns & -0.046 & ns \\
\hline RVESVI $\left(\mathrm{mL} / \mathrm{m}^{2}\right)$ & 0.313 & ns & -0.166 & ns \\
\hline QRS (ms) & -0.235 & ns & -0.069 & ns \\
\hline \multicolumn{5}{|l|}{ Vorticity } \\
\hline$\omega_{\text {RVOT-Diastole }}$ & -0.434 & ns & -0.5497 & 0.016 \\
\hline RVOT-VQ ( $\left.\omega_{\text {RVOT-Diastole }} / \omega_{\text {RVOT-Systole }}\right)$ & -0.587 & 0.008 & -0.678 & 0.001 \\
\hline RV-DVQ ( $\left.\omega_{\text {RVOT-Diastole }} / \omega_{\text {RV Inflow-Diastole }}\right)$ & -0.266 & Ns & -0.641 & 0.003 \\
\hline \multicolumn{5}{|l|}{ Energy Loss } \\
\hline VEL $L_{\text {RVOT-Diastole }}$ & -0.497 & 0.03 & -0.375 & ns \\
\hline RVOT-EQ (VEL & -0.617 & 0.005 & -0.6902 & 0.001 \\
\hline
\end{tabular}

be optimized to prevent long-term, irreversible consequences of RV dysfunction such as ventricular arrhythmias, exercise intolerance and overt systolic dysfunction [6, 29-31]. To date the International Multicenter TOF Registry (INDICATOR), a multi-center international cohort study, provides the best interpretation of RV dysfunction in rTOF patients by CMR, identifying RVEF as a significant risk factor for death and ventricular tachycardia [32]. However, it is also clear that conventional metrics of PR\% and ventricular size/function cannot predict deterioration of the RV [4]. It has also been shown by meta-analysis that for large RVs, improvements in RV size after PVR do not necessarily improve RVEF and is less correlated to improvement of symptoms [33]. Electromechanical dyssynchrony and scar burden appear to be more associated with exercise intolerance and RVEF compared to PR\% [10, 34]. The CMR metrics do not fully capture the underlying pathophysiology of RV dysfunction in rTOF patients. Thus, PR and RVEDVI are only fairly correlated with RVEF and not correlated with exercise capacity. Meanwhile, the proposed 4D flow parameters have correlations with RVEF, RF\%, RVEDVI, and exercise capacity.

The 4D flow parameters and analysis of diastolic vortical flow patterns show that RV dilation patients still have preserved diastolic filling, despite having larger RV size. In control and RV dilation cases, the observed "donut" of diastolic vortex from tricuspid inflow is consistent with our previous in-vitro investigations [18-20], computational studies derived from 3D echocardiography [35-37] and in-vivo observations of 4D flow [38]. Early work by Pasipoularides et al. demonstrated the role of the diastolic vortex in reducing the "convective deceleration load" and improve ventricular filling [36]. Specifically, 

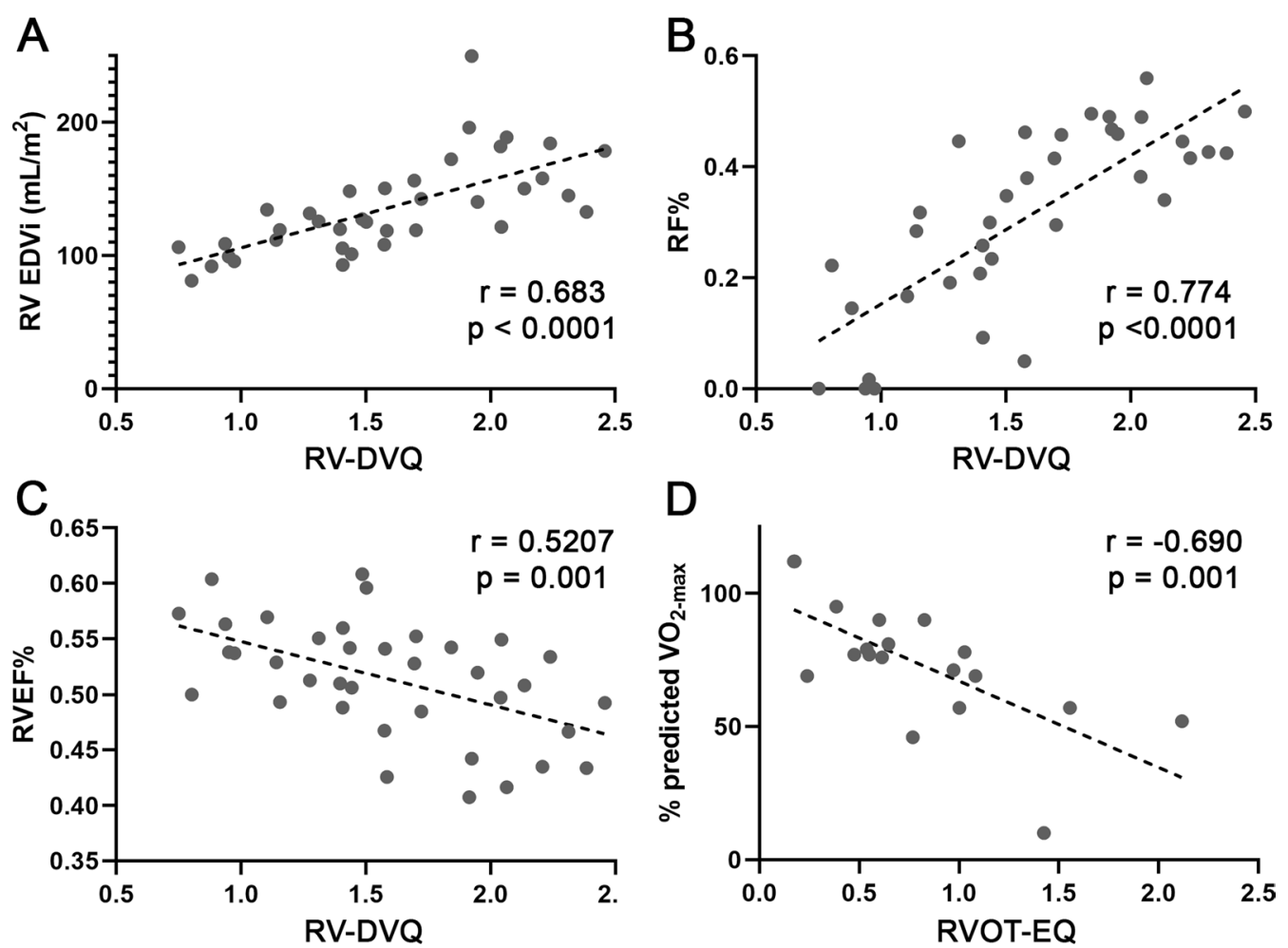

Fig. 6 Correlations of vorticity and energy loss to conventional indices in subgroup analysis. A-C are from subgroup analysis of repaired tetralogy of Fallot (rTOF) patients with previous anatomy of Tetralogy of Fallot, Pulmonary stenosis (TOF-PS) and only infundibular or transannular patch repair (i.e. no conduit placement) and no further surgical reintervention (i.e. no pulmonary valve replacement). D is from subgroup analysis of RTOF patients with exercise stress test

the "donut" vortex is likely generated by the longitudinal motion of the RV during diastole, with no active acceleration/deceleration components that would exert additional hemodynamic forces in the RV. The propagation of the inflow vortex towards the RVOT may also facilitate systolic flow [39]. RV-DVQ takes into account the relative impact of the inflow vortex towards the RVOT; thus as a normalized parameter, RV-DVQ is not significantly different between RV dilation and normal controls (Fig. 4B).

In our study, specific regional measurement by $4 \mathrm{D}$ flow was key to highlighting the complex interplay between PR and RV size/function in rTOF patients. In-vivo and in-vitro studies of aortic regurgitation in the left ventricle show that when the inflow vortex is interrupted by a colliding jet of insufficiency, there is increased vortical flow formation and energy loss [40-42]. rTOF patients in similar fashion have higher vorticity and energy loss from PR in the RVOT, as the local jet collision creates a distinct intracardiac topology which generates shear layers, small-scale turbulence or turbulent-like flow [16, 18-20]. This effect is determined by PR, inflow, RV geometry (e.g. the relative angle of inflow and PR), which may explain why
RVOT vorticity and energy loss correlate with RV size, RV function and PI in RTOF. Considering that RV function and exercise capacity tend to be preserved in rTOF with transatrial-transpulmonary approach, or in patients with isolated PS status post valvotomy (i.e. minimized or absent ventriculotomy in RVOT) [43, 44], the RVOT may also function to attenuate the abnormal flow features from PR.

Furthermore, the native diastolic vortex from tricuspid inflow is dominated by RVOT vorticity, leading to higher RV-DVQ (Fig. 4B). RV-DVQ was also correlated with longer QRS duration (i.e. right bundle branch block). Electromechanical dyssynchrony likely affected RV longitudinal wall motion [10], which in turn weakens the tricuspid inflow vortex. The competing nature between PI and tricuspid inflow is also observed in in-vitro studies $[16,18]$, and may lead to abnormal hemodynamic forces in directed from the RVOT towards the RV base, as demonstrated by Sjoberg et al. [45]. The vortex interaction and resultant hemodynamic forces likely contributes to altered mechanotransductive environment that leads to RV dysfunction [46, 47]. The abnormal hemodynamic forces may also contribute to the abnormal RVOT 
shape geometry found in larger RVs [7]. PVR would likely restore the natural diastolic vortex and help restore RV function; thus RVOT vorticity and energy loss could potentially be biomarkers to guide PVR therapy.

The subgroup correlation results of RV-DVQ, RVOTVQ and RVOT-EQ suggest that the $4 \mathrm{D}$ flow measurements, by capturing PR as localized intracardiac phenomena, reflects the clinical range of RV dilation and dysfunction created in rTOF patients. Shibata et al. using vector flow mapping by echocardiography, demonstrated that diastolic energy losses correlated with RV deterioration [48]. Measurements of kinetic energy have also demonstrated global RV abnormalities with varying correlations to RV size and RV function [15, 49]. Frediksson et al. in particular measured turbulent kinetic energy [14] derived from the magnitude imaging of individual flow-encoding segments rather than the velocity field. In that study, rTOF patients with larger RVEDVI had higher turbulent kinetic energy. These abnormalities are likely sources of energy loss that contributes to RV pump inefficiency in rTOF patients $[48,50]$.

Furthermore, the interaction between PR and tricuspid inflow potentially limits augmentation of RV cardiac output during exercise, leading to exercise intolerance. Previous CMR studies have not demonstrated any significant correlation between RV size and PR with exercise capacity $[8,9,11]$, informing the use of modified parameters such as ventricular global function index [9]. Thus far, only right ventricular systolic dysfunction and branch pulmonary artery size discrepancy have correlated with decreased exercise capacity $[7,11,51]$. In our subgroup cohort, RVOT-VQ and RVOT-EQ correlated with $\mathrm{VO}_{2-\max }$ and $\%$ predicted $\mathrm{VO}_{2-\max }$, suggesting that the abnormal intracardiac flow patterns from PR is still the link to exercise intolerance in rTOF patients. Ideally, these 4D flow parameters would be investigated with exercise CMR [52], although the decreased signalto-noise ratio and motion artifact during exercise would likely limit interpretation of such findings. We aim to develop these as novel imaging biomarkers linking the pathophysiology between PR and exercise intolerance, to ultimately assist in the decision of optimal timing of PVR.

In addition to intracardiac flow, there are other potential biomarkers of RV size/function beyond volumetric measurements. Studies investigating myocardial strain by feature tracking have demonstrated impairments in $\mathrm{RV}$ global strain and ventricular dyssynchrony in rTOF patients [30, 53]. RV scar burden as defined by late gadolinium enhancement volume also correlates with ventricular dysfunction and risk of inducible ventricular arrhythmias [34]. Shape modeling studies have demonstrated morphological changes beyond measurements of RVEDVI and RVESVI [5, 7, 54]. Computational load analysis of the RV myocardium, including the akinetic RVOT free wall [55] may also be sensitive in predicting RV dysfunction.

\section{Prior studies}

Compared to other 4D CMR studies on rTOF, our study has the benefit of (1) a larger sample size, with comparison against normal controls as well as RVD representing other chronic volume loading states and (2) a specific regional focus of $4 \mathrm{D}$ flow biomarkers to avoid dilution of results by including tricuspid inflow. For example, Hirtler et al. investigated 24 rTOF patients and measured higher mean RV vorticity, but these measurements were paradoxically negatively correlated with RV size [12]. This finding likely resulted from measurements derived in the 4-chamber cine plane, which only isolates the tricuspid inflow vortex (whereas our data would suggest that this component is weakened by PR).

\section{Study limitations}

The study, while larger than other 4D flow studies of rTOF, is still a single-center analysis and limited by sample size. The rTOF cohort was heterogenous in both anatomy and surgical repair, limiting the correlations observed in the study (although subgroup analysis of TOF-PS patients still had consistent results). As a retrospective study, the clinical $4 \mathrm{D}$ flow datasets did not include dedicated magnitude imaging which is required to calculate other 4D flow-based parameters such as turbulent kinetic energy. Late gadolinium enhancement was also not routinely performed in this study, which precluded the investigation of peri-patch fibrosis in the RVOT [34] and its effect on intracardiac flow. For 4D flow analysis, we intentionally cut arbitrary, static planes in RV end-diastolic models to minimize inter-observer variability inherent with mask segmentation of RV regions. This potentially limited analysis of the RVOT during systolic movement, although this effect may be minimal as rTOF cases tend to have akinetic RVOT. There was inconsistent timing of exercise stress test in rTOF patients, although the time interval between CMR and stress tests were still within recommended surveillance guidelines of rTOF patients [2] and is unlikely to significantly alter results based on previous longitudinal studies [56, 57]. Most importantly, the limited spatial and temporal resolution of $4 \mathrm{D}$ flow prohibited detailed assessment of smaller vortical interactions formed by the collision of PR into the tricuspid vortex and the propagation of turbulent flow into the RV body and myocardium, particularly the trabeculations [58]. Thus, we did not assess hemodynamic parameters such as wall shear stress along the RV free wall, which likely has an influence on RV dilation. 


\section{Future studies}

Future work will focus on in-depth flow quantification in rTOF patients, to further elaborate on the influence of vorticity/energy loss towards RV dysfunction and exercise intolerance. These will include refining computational models of RV intracardiac flow in rTOF patients [18-20] and using advanced echocardiography techniques such as vector flow mapping [48] or blood speckle imaging [59]. These methods could potentially overcome the spatial and temporal limitations of 4D flow, further refining flow biomarkers to account for other factors such as heart rate, voxel size or presence of late gadolinium enhancement. As the variable RVOT geometry in rTOF patients [7] may also alter the angle of jet collision between PI and tricuspid inflow, we will also investigate this relationship with statistical shape modeling. We plan to prospectively use 4D flow and computational modelling to investigate intracardiac flow in RTOF patients before-and-after PVR, as well as during exercise. We aim to further develop vorticity and energy loss as clinical biomarkers to aid in timing of PVR for rTOF patients.

\section{Conclusion}

In rTOF patients, abnormal vorticity and energy loss dominate the RVOT compared to tricuspid inflow. These 4D flow-based parameters are potential biomarkers that are distinct from other forms of chronic RV volume loading, representing abnormal intracardiac flow that contribute to RV dysfunction and exercise intolerance. Future studies should investigate the role of intracardiac 4D flow in guiding PVR therapy.

\section{Abbreviations}

3D: Three dimensional; ASD: Atrial septal defect; CMR: Cardiovascular magnetic resonance; DORV: Double outlet right ventricle; MRA: Magnetic resonance angiography; PAPVR: Partial anomalous pulmonary venous return; PR: Pulmonary regurgitation; PR\%: Pulmonary regurgitant fraction; PS: Pulmonic Stenosis; PVR: Pulmonary valve replacement; rTOF: Repaired Tetralogy of Fallot; RV: Right ventricle/right ventricular; RV-DVQ: Right ventricular diastolic vorticity quotient; RV-PA: Right ventricle-to-pulmonary artery conduit repair; RVD: Right ventricular dilation; RVEDVI: Right ventricular end-diastolic volume index; RVEF: Right ventricular ejection fraction; RVESVI: Right ventricular end-systolic volume index; RVOT: Right ventricular outflow tract; RVOT-DVQ: Right ventricular outflow tract diastolic vorticity quotient; RVOT-EQ: Right ventricular outflow tract energy quotient; RVOT-VQ: Right ventricular outflow tract vorticity quotient; SAx: Short axis; SSFP: Steady state free precession; TOFPA: Tetralogy of Fallot with pulmonary atresia; TOF-PS: Tetralogy of Fallot with pulmonary stenosis; VEL: Viscous energy loss.
\end{abstract}

\section{Acknowledgements}

The authors would like to acknowledge the support provided by all members of the CMR program at Children's National Hospital.

\section{Authors' contributions}

YHL was responsible for the study design, analysis of CMR measurements, interpretation of results and wrote the manuscript. VC and JM contributed to analysis of CMR measurements and writing the manuscript. FC and EB contributed to the interpretation of results and writing the manuscript. LO contributed to the study design, interpretation of results and writing the manuscript. All authors read and approved the final manuscript.

\section{Funding}

Dr. Yue-Hin Loke receives partial salary support from NIH R01 HL143468-01 and R21 HL156045. Dr. Francesco Capuano also received support from NIH UL1TR001876.

This publication was supported by Award Number UL1TR001876 from the NIH National Center for Advancing Translational Sciences. Its contents are solely the responsibility of the authors and do not necessarily represent the official views of the National Center for Advancing Translational Sciences or the National Institutes of Health. This work was also supported by institutional funding through Children's National Hospital (Board of Visitors grant) to pay for licensing of segmentation software (Mimics, Materialise). Dr. Francesco Capuano was supported by Università degli Studi di Napoli "Federico II".

\section{Availability of data and materials}

The datasets used and/or analyzed during the current study are available from the corresponding author on reasonable request.

\section{Declarations}

\section{Ethics approval and consent to participate}

This retrospective study was approved by the Institutional Review Board. Waiver of consent was obtained. All procedures followed were in accordance with the ethical standards of the responsible committee on human experimentation (institutional and national) and with the Helsinki Declaration of 1975 , as revised in 2000

\section{Consent for publication}

Not applicable.

\section{Competing interests}

The authors declare they have no competing interests.

\section{Author details}

${ }^{1}$ Present Address: Division of Cardiology, Children's National Medical Center, 111 Michigan Ave NW, W3-200, Washington, DC 20010, USA. Department of Mechanics, Mathematics and Management, Polytechnic University of Bari, Bari, Italy. ${ }^{3}$ Sheikh Zayed Institute for Pediatric Surgical Innovation, Children's National Medical Center, 111 Michigan Ave NW, Washington, DC 20010, USA. ${ }^{4}$ Department of Mechanical and Aerospace Engineering, George Washington University, Washington, DC 20052, USA.

Received: 16 March 2021 Accepted: 28 June 2021

Published online: 19 August 2021

\section{References}

1. Bhatt AB, Foster E, Kuehl K, Alpert J, Brabeck S, Crumb S, et al. Congenital heart disease in the older adult: a scientific statement from the American Heart Association. Circulation. 2015;131(21):1884-931.

2. Stout KK, Daniels CJ, Aboulhosn JA, Bozkurt B, Broberg CS, Colman JM, et al. 2018 AHA/ACC guideline for the management of adults with congenital heart disease: a report of the American College of Cardiology/ American Heart Association task force on clinical practice guidelines. Circulation. 2019;139:14. https://doi.org/10.1161/CIR.0000000000000603.

3. Geva T. Indications for pulmonary valve replacement in repaired Tetralogy of Fallot: the quest continues. Circulation. 2013;128(17):1855-7.

4. Wald RM, Valente AM, Gauvreau K, Babu-Narayan SV, Assenza GE, Schreier J, et al. Cardiac magnetic resonance markers of progressive RV dilation and dysfunction after tetralogy of Fallot repair. Heart. 2015;101(21):1724-30.

5. Zaidi SJ, Cossor W, Singh A, Maffesanti F, Kawaji K, Woo J, et al. Threedimensional analysis of regional right ventricular shape and function in repaired tetralogy of Fallot using cardiovascular magnetic resonance. Clin Imaging. 2018;52:106-12.

6. Heng EL, Gatzoulis MA, Uebing A, Sethia B, Uemura H, Smith GC, et al. Immediate and midterm cardiac remodeling after surgical pulmonary 
valve replacement in adults with repaired Tetralogy of Fallot: a prospective cardiovascular magnetic resonance and clinical study. Circulation. 2017:136(18):1703-13.

7. Shen W-C, Chen C-A, Chang C-I, Chen Y-S, Huang S-C, Wu M-H, et al. Outflow tract geometries are associated with adverse outcome indicators in repaired tetralogy of Fallot. J Thoracic Cardiovasc Surg. 2020;225:6702.

8. Avesani M, Borrelli N, Krupickova S, Sabatino J, Donne GD, Ibrahim A, et al. Echocardiography and cardiac magnetic resonance in children with repaired tetralogy of Fallot: new insights in cardiac mechanics and exercise capacity. Int J Cardiol. 2020;321:144-9.

9. Ta HT, Critser PJ, Alsaied T, Germann J, Powell AW, Redington AN, et al. Modified ventricular global function index correlates with exercise capacity in repaired Tetralogy of Fallot. J Am Heart Assoc. 2020;9(14):e016308.

10. Lumens J, Fan CS, Walmsley J, Yim D, Manlhiot C, Dragulescu A, et al. Relative impact of right ventricular electromechanical dyssynchrony versus pulmonary regurgitation on right ventricular dysfunction and exercise intolerance in patients after repair of Tetralogy of Fallot. J Am Heart Assoc. 2019;8:2. https://doi.org/10.1161/JAHA.118.010903.

11. Rashid I, Mahmood A, Ismail TF, O'Meagher S, Kutty S, Celermajer D, et al. Right ventricular systolic dysfunction but not dilatation correlates with prognostically significant reductions in exercise capacity in repaired Tetralogy of Fallot. Eur Heart J Cardiovasc Imag. 2020;21(8):906-13.

12. Hirtler D, Garcia J, Barker AJ, Geiger J. Assessment of intracardiac flow and vorticity in the right heart of patients after repair of tetralogy of Fallot by flow-sensitive 4D MRI. Eur Radiol. 2016;26(10):3598-607.

13. Francois CJ, Srinivasan S, Schiebler ML, Reeder SB, Niespodzany E, Landgraf BR, et al. 4D cardiovascular magnetic resonance velocity mapping of alterations of right heart flow patterns and main pulmonary artery hemodynamics in tetralogy of Fallot. J Cardiovasc Magn Reson. 2012;14(1):16.

14. Fredriksson A, Trzebiatowska-Krzynska A, Dyverfeldt P, Engvall J, Ebbers T, Carlhäll C-J. Turbulent kinetic energy in the right ventricle: Potential MR marker for risk stratification of adults with repaired Tetralogy of Fallot. J Magn Reson Imaging. 2018;47(4):1043-53.

15. Robinson JD, Rose MJ, Joh M, Jarvis K, Schnell S, Barker AJ, et al. 4-D flow magnetic-resonance-imaging-derived energetic biomarkers are abnormal in children with repaired tetralogy of Fallot and associated with disease severity. Pediatr Radiol. 2019;49(3):308-17.

16. Mikhail A, Labbio GD, Darwish A, Kadem L. How pulmonary valve regurgitation after tetralogy of fallot repair changes the flow dynamics in the right ventricle: An in vitro study. Med Eng Phys. 2020:83:48-55.

17. Moceri P, Duchateau N, Gillon S, Jaunay L, Baudouy D, Squara F, et al. Three-dimensional right ventricular shape and strain in congenital heart disease patients with right ventricular chronic volume loading. Eur Heart J. 2020;5:jeaa189.

18. Capuano F, Loke YH, Olivieri L, Balaras E. Computational modeling of right ventricle flow dynamics in congenital heart disease. In: García-Villalba M, Kuerten H, Salvetti MV, editors. Direct and large eddy simulation XII. Cham: Springer; 2020. p. 477-83.

19. Capuano F, Romano L, Loke YH, Dellegrottaglie S, Notorio M, Cocchia R, et al. P1449 CMR-driven computational modeling of right ventricular flow dynamics. Eur Heart J. 2020;21(1):jez319.877.

20. Loke YH, Capuano F, Balaras E, Olivieri L. Computational modeling of right ventricular motion and intracardiac flow in repaired tetralogy of fallot. Cardiovasc Eng Tech. 2021. https://doi.org/10.1007/ s13239-021-00558-3.

21. Loke YH, Krieger A, Sable C, Olivieri L. Novel uses for three-dimensional printing in congenital heart disease. Curr Pediatr Rep. 2016;4(2):28-34.

22. Loke YH, Harahsheh AS, Krieger A, Olivieri L. Usage of 3D models of tetralogy of Fallot for medical education: impact on learning congenital heart disease. BMC Med Educ. 2017;17:124. https://doi.org/10.1186/ s12909-017-0889-0.

23. Wald RM, Haber I, Wald R, Valente AM, Powell AJ, Geva T. Effects of regional dysfunction and late gadolinium enhancement on global right ventricular function and exercise capacity in patients with repaired tetralogy of Fallot. Circulation. 2009;119(10):1370-7.

24. Nakaji K, Itatani K, Tamaki N, Morichi H, Nakanishi N, Takigami M, et al. Assessment of biventricular hemodynamics and energy dynamics using lumen-tracking 4D flow MRI without contrast medium. J Cardiol. 2021;914:508

25. Cibis M, Jarvis K, Markl M, Rose M, Rigsby C, Barker AJ, et al. The effect of resolution on viscous dissipation measured with $4 \mathrm{D}$ flow MRl in patients with Fontan circulation: evaluation using computational fluid dynamics. J Biomech. 2015;48(12):2984-9.

26. Mercer-Rosa L, Yang W, Kutty S, Rychik J, Fogel M, Goldmuntz E. Quantifying pulmonary regurgitation and right ventricular function in surgically repaired Tetralogy Of Fallot: a comparative analysis of echocardiography and magnetic resonance imaging. Circ Cardiovasc Imaging. 2012;5(5):637-43.

27. Bhat M, Goldmuntz E, Fogel MA, Rychik J, Mercer-Rosa L. Longitudinal validation of the diastolic to systolic time-velocity integral ratio as a Doppler-derived measure of pulmonary regurgitation in patients with repaired Tetralogy of Fallot. Pediatr Cardiol. 2017;38(2):240-6.

28. Mukaka MM. Statistics corner: A guide to appropriate use of correlation coefficient in medical research. Malawi Med J. 2012;24(3):69-71.

29. Waldmann V, Bouzeman A, Duthoit G, Koutbi L, Bessiere F, Labombarda F, et al. Long-term follow-up of patients with Tetralogy of Fallot and implantable cardioverter defibrillator: The DAI-T4F nationwide registry. Circulation. 2020;142(17):1612-22.

30. Balasubramanian S, Harrild DM, Kerur B, Marcus E, del Nido P, Geva T, et al. Impact of surgical pulmonary valve replacement on ventricular strain and synchrony in patients with repaired tetralogy of Fallot: a cardiovascular magnetic resonance feature tracking study. J Cardiovasc Magn Reson. 2018;20(1):37.

31. O'Byrne ML, Glatz AC, Mercer-Rosa L, Gillespie MJ, Dori Y, Goldmuntz $E$, et al. Trends in pulmonary valve replacement in children and adults with tetralogy of fallot. Am J Cardiol. 2015;115(1):118-24.

32. Preoperative Predictors of Death and Sustained Ventricular Tachycardia After Pulmonary Valve Replacement in Patients With Repaired Tetralogy of Fallot Enrolled in the INDICATOR Cohort. 10.

33. Ferraz Cavalcanti PE, Sá MPBO, Santos CA, Esmeraldo IM, de Escobar RR, de Menezes AM, et al. Pulmonary valve replacement after operative repair of Tetralogy of Fallot. J Am Coll Cardiol. 2013;62(23):2227-43.

34. Ghonim S, Ernst S, Keegan J, Giannakidis A, Spadotto V, Voges I, et al. Three-dimensional late gadolinium enhancement cardiovascular magnetic resonance predicts inducibility of ventricular tachycardia in adults with repaired Tetralogy of Fallot. Circ Arrhythm Electrophysiol. 2020;13:11. https://doi.org/10.1161/CIRCEP.119.008321.

35. Pasipoularides A, Shu M, Shah A, Womack MS, Glower DD. Diastolic right ventricular filling vortex in normal and volume overload states. Am J Physiol Heart Circ Physiol. 2003;284(4):H1064-72.

36. Pasipoularides A. Evaluation of right and left ventricular diastolic filling. J of Cardiovasc Trans Res. 2013;6(4):623-39.

37. Mangual JO, Domenichini F, Pedrizzetti G. Describing the highly three dimensional right ventricle flow. Ann Biomed Eng. 2012;40(8):1790-801.

38. Fredriksson AG, Zajac J, Eriksson J, Dyverfeldt P, Bolger AF, Ebbers T, et al. 4-D blood flow in the human right ventricle. Am J Physiol Heart Circ Physiol. 2011;301(6):H2344-50.

39. Mangual JO, Domenichini F, Pedrizzetti G. Three dimensional numerical assessment of the right ventricular flow using $4 \mathrm{D}$ echocardiography boundary data. Eur J Mech B Fluids. 2012;35:25-30.

40. Stugaard M, Koriyama H, Katsuki K, Masuda K, Asanuma T, Takeda Y, et al. Energy loss in the left ventricle obtained by vector flow mapping as a new quantitative measure of severity of aortic regurgitation: a combined experimental and clinical study. Eur Heart J Cardiovasc Imag. 2015;16(7):723-30.

41. Pedrizzetti G, Sengupta PP. Vortex imaging: new information gain from tracking cardiac energy loss. European Heart Journal. 2015;16(7):719-20.

42. Okafor I, Raghav V, Condado JF, Midha PA, Kumar G, Yoganathan AP. Aortic regurgitation generates a kinematic obstruction which hinders left ventricular filling. Ann Biomed Eng. 2017;45(5):1305-14.

43. Ye XT, Buratto E, Konstantinov IE, d'Udekem Y. Does transatrial-transpulmonary approach improve outcomes compared with transventricular approach in non-neonatal patients undergoing tetralogy of Fallot repair? Interact Cardiovasc Thorac Surg. 2019;29(6):960-6.

44. Zdradzinski MJ, Qureshi AM, Stewart R, Pettersson G, Krasuski RA. Comparison of long-term postoperative sequelae in patients with tetralogy of Fallot versus isolated pulmonic stenosis. Am J Cardiol. 2014;114(2):300-4.

45. Sjöberg P, Töger J, Hedström E, Arvidsson P, Heiberg E, Arheden H, et al. Altered biventricular hemodynamic forces in patients with repaired tetralogy of Fallot and right ventricular volume overload 
because of pulmonary regurgitation. Am J Physiol Heart Circ Physiol. 2018;315(6):H1691-702.

46. Pasipoularides A. Mechanotransduction mechanisms for intraventricular diastolic vortex forces and myocardial deformations: part 1. J of Cardiovasc Trans Res. 2015;8(1):76-87.

47. Pasipoularides A. Mechanotransduction Mechanisms for Intraventricular Diastolic Vortex Forces and Myocardial Deformations: Part 2. J Cardiovasc Trans Res. 2015;8(5):293-318.

48. Shibata M, Itatani K, Hayashi T, Honda T, Kitagawa A, Miyaji K, et al. Flow energy loss as a predictive parameter for right ventricular deterioration caused by pulmonary regurgitation after Tetralogy of Fallot repair. Pediatric Cardiology. 2018. https://doi.org/10.1007/s00246-018-1813-z.

49. Jeong D, Anagnostopoulos PV, Roldan-Alzate A, Srinivasan S, Schiebler $\mathrm{ML}$, Wieben $\mathrm{O}$, et al. Ventricular kinetic energy may provide a novel noninvasive way to assess ventricular performance in patients with repaired tetralogy of Fallot. J Thorac Cardiovasc Surg. 2015;149(5):1339-47.

50. Fogel MA, Sundareswaran KS, de Zelicourt D, Dasi LP, Pawlowski T, Rome J, et al. Power loss and right ventricular efficiency in patients after Tetralogy of Fallot repair with pulmonary insufficiency: clinical implications. J Thorac Cardiovasc Surg. 2012:143(6):1279-85.

51. Meadows J, Powell AJ, Geva T, Dorfman A, Gauvreau K, Rhodes J. Cardiac magnetic resonance imaging correlates of exercise capacity in patients with surgically repaired Tetralogy of Fallot. Am J Cardiol. 2007;100(9):1446-50.

52. Macdonald JA, Beshish AG, Corrado PA, Barton GP, Goss KN, Eldridge MW, et al. Feasibility of cardiovascular four-dimensional flow MRI during exercise in healthy participants. Radiol Cardiothorac Imaging. 2020;2(3):e190033.

53. Berganza FM, de Alba CG, Özcelik N, Adebo D. Cardiac magnetic resonance feature tracking biventricular two-dimensional and three-dimensional strains to evaluate ventricular function in children after repaired Tetralogy of Fallot as compared with healthy children. Pediatr Cardiol. 2017:38(3):566-74

54. Leonardi B, Taylor AM, Mansi T, Voigt I, Sermesant M, Pennec X, et al. Computational modelling of the right ventricle in repaired tetralogy of Fallot: can it provide insight into patient treatment? Eur Heart J. 2013:14(4):381-6.

55. Tang D, del Nido PJ, Yang C, Zuo H, Huang X, Rathod RH, et al. Patientspecific MRI-based right ventricle models using different zero-load diastole and systole geometries for better cardiac stress and strain calculations and pulmonary valve replacement surgical outcome predictions. PLOS ONE. 2016;11(9):e0162986.

56. Bhat M, Mercer-Rosa L, Fogel MA, Harris MA, Paridon SM, McBride MG, et al. Longitudinal changes in adolescents with TOF: implications for care. Eur Heart J Cardiovasc Imaging. 2017;18(3):356-63.

57. Kipps AK, Graham DA, Harrild DM, Lewis E, Powell AJ, Rhodes J. Longitudinal exercise capacity of patients with repaired tetralogy of fallot. Am J Cardiol. 2011;108(1):99-105.

58. Sacco F, Paun B, Lehmkuhl O, lles TL, laizzo PA, Houzeaux G, et al. Evaluating the roles of detailed endocardial structures on right ventricular haemodynamics by means of CFD simulations. Int J Numer Method Biomed Eng. 2018;34(9):e3115.

59. Nyrnes SA, Fadnes S, Wigen MS, Mertens L, Lovstakken L. Blood speckletracking based on high-frame rate ultrasound imaging in pediatric cardiology. J Am Soc Echocardiogr. 2020;33(4):493-503.e5.

\section{Publisher's Note}

Springer Nature remains neutral with regard to jurisdictional claims in published maps and institutional affiliations.
Ready to submit your research? Choose BMC and benefit from:

- fast, convenient online submission

- thorough peer review by experienced researchers in your field

- rapid publication on acceptance

- support for research data, including large and complex data types

- gold Open Access which fosters wider collaboration and increased citations

- maximum visibility for your research: over $100 \mathrm{M}$ website views per year

At BMC, research is always in progress.

Learn more biomedcentral.com/submissions 